\title{
Vacilaciones y planificación discursiva en entrevistas de la Asamblea Legislativa del Distrito Federal: la elección de la ombudsperson de derechos humanos en 2013
}

\author{
Luis Bernardo Quesada Nieto* \\ The Graduate Center, CUNY, Estados Unidos
}

\begin{abstract}
Resumen
El presente trabajo estudia seis tipos de fenómenos orales identificados como vacilaciones (sonidos breves, repeticiones no intencionales, frases cortadas, palabras cortadas, sonidos largos y alargamientos de palabra), en un corpus compuesto por entrevistas realizadas en la Comisión de Derechos Humanos de la VI Legislatura de la Asamblea Legislativa del Distrito Federal (hoy Congreso de la Ciudad de México) en 2013. Estas entrevistas fueron diseñadas y conducidas por legisladores de esta Comisión con el propósito de elegir de entre un grupo de ciudadanos al "mejor" para ocupar el cargo de ombudsperson de la Comisión de Derechos Humanos de la ciudad. Luego de proponer una definición de vacilación, se plantea que, así como el tipo de vacilaciones y su frecuencia de aparición muestran patrones de variación distintos de una secuencia discursiva a otra en las entrevistas, estos patrones también están estrechamente relacionados con el grado de planificación que muestra el discurso. A partir de esta correlación de variables se propone así
\end{abstract}

\footnotetext{
* Para correspondencia, dirigirse a: Luis Bernardo Quesada Nieto (lquesadanieto@ gradcenter.cuny.edu).
} 
una clasificación de fenómenos según las funciones discursivas de estos en la interacción.

Palabras clave: vacilaciones, planificación discursiva, entrevista, discurso parlamentario, ombudsperson, Congreso de la Ciudad de México.

\title{
Hesitation Markers and Discursive Planning in Interviews within MeXico City's Local Congress: the 2013 Human Rights OMBUdSPERSON EleCtion
}

\begin{abstract}
This article analyses six types of hesitation markers (short fillers, non-intentional repetitions, unfinished phrases, unfinished words, long fillers, and word lengthening), identified in a corpus of interviews designed by the Comisión de Derechos Humanos of the $6^{\text {th }}$ Asamblea Legislativa del Distrito Federal (today Mexico City's Congress) in 2013. These interviews were carried out by representatives ascribed to this legislative entity and its purpose was to find "the best" candidate among a group of citizens, to fill the ombudsperson position at the city's Human Rights local office. After presenting a definition of hesitation marker, I argue that the type of hesitation marker and its frequency show variation patterns that change from one discursive sequence to the other within the interviews; these patterns are also closely related to the discourse planning degree. From this variable correlation, I propose a classification of phenomena based on the discursive functions they have in interaction.
\end{abstract}

Keywords: hesitation markers, discourse planning, interview, parliamentary discourse, ombudsperson, Mexico City's Congress.

Recibido: 27/12/17

Aceptado: 18/01/19 


\section{LAS VACILACIONES Y EL DISCURSO PARLAMENTARIO ${ }^{1}$}

La investigación sobre fenómenos de vacilación en español ha sido y es hasta la fecha escasa. Un acercamiento a estos fenómenos resulta relevante por una serie de motivos, entre los que destacan: 1) ser un posible reflejo de la arraigada tradición logocéntrica que opera en situaciones sociales en las que se busca acercar la lengua oral a la lengua escrita; 2) ser marcas del proceso cognitivo del hablante en la enunciación; 3) servir como apoyos o estrategias orales de cohesión argumentativa del discurso; 4) ser indicadores de variación diastrática y diafásica, entre otras ${ }^{2}$.

Este trabajo, que investiga las funciones conversacionales de las vacilaciones, busca contribuir a la explicación de ciertos procesos de elaboración discursiva que aparecen en el uso de la lengua en una situación interactiva formal y protocolar, en la Asamblea Legislativa del Distrito Federal (ALDF-Ciudad de México). Se pretende en concreto aportar a la comprensión estos fenómenos en la lengua española, en un contexto de habla ordinario al interior del proceso legislativo de esta institución. El camino que se sigue comienza con una propuesta definitoria de la categoría "vacilación", la presentación de algunos fenómenos que dicha definición abarca según aparecen en el corpus y, finalmente, una identificación y categorización según las funciones discursivas que presentan.

Esta investigación se ubica en el ámbito del discurso parlamentario mexicano, que ha sido analizado por Carbó (1987) y Vázquez Laslop (2011). Ambas contribuciones han partido de eventos discursivos que se dan en el Pleno del recinto legislativo federal, particularmente en los debates en tribuna que anteceden a las votaciones legislativas. El discurso parlamentario, no obstante, contiene otras variedades de "interacción institucionalizada", como es el caso del trabajo que realizan las comisiones legislativas, que no han sido abordadas desde la sociolingüística.

La atención "científica" dirigida a distintos fenómenos de la oralidad identificados como vacilaciones comenzó a darse a partir de mediados del siglo XX, desde campos como la psicología (Mahl 1987) y las ciencias

Agradezco a Dora Pellicer (ENAH) y a María Eugenia Vázquez Laslop (Colmex) por sus orientaciones durante el proceso de investigación, así como a Leonor Orozco (UNAM), quien además hizo sugerencias al presente texto. Gracias también a José del Valle (CUNY) por su lectura y comentarios adicionales.

2 Estos motivos para el estudio de las vacilaciones se enumeran aquí como supuestos sobre los cuales se justifica la profundización sobre el tema en futuras investigaciones. 
cognitivas (Dalton y Hardcastle 1977; Goldman-Eisler 1972; Reynolds y Paivio 1968). En el ámbito lingüístico han aparecido acercamientos que resaltan el rol comunicativo y conversacional de las vacilaciones (Clark 1994; Clark y Fox Tree 2002; Fox Tree 1995; Levelt 1983; Schegloff et al. 1977; Tannen 1987) así como trabajos sobre aspectos léxicos (Schachter et al. 1991), sintácticos (Watanabe et al. 2008), acústicos (Duez 2001b) y prosódicos (Brennan y Williams 1995). Asimismo, otros autores (Ávila 1999; Bortfeld et al. 2001; Duez 2001; Laforest 1997; Schober 2006; Vasilescu y AddaDecker 2006) las han estudiado desde enfoques interactivos y contextuales. Más recientemente, destacan los trabajos de Levinson y Torreira (2015) y de Crible (2018).

A pesar del interés que ha suscitado la vacilación en la lingüística y otras disciplinas especialmente a partir de la década de los 70, en el ámbito hispánico ha recibido una atención relativamente escasa. Un estudio pionero sobre las vacilaciones en México es el de Ávila (1999), quien las analizó en entrevistas radiofónicas. Ávila se enfocó en tres tipos: pausas oralizadas, repeticiones y rupturas sintácticas, que corresponden a los tres tipos de vacilación (hésitation) propuestos por Laforest (1997), y encontró que, aunque el género de entrevista radiofónica "se perciba como una unidad desde la dimensión comunicativa, carece de una constitución monolítica de una sola pieza y su construcción es un proceso gradual [que se da] con el auxilio de puentes que unen trozos o fragmentos enunciativos" (p. 71). En este sentido, para Ávila las vacilaciones son "una especie de aglutinantes discursivos" (p. 72).

Destaca también la aportación de Menjura (2007), quien reconoce que fenómenos orales como las repeticiones, los silencios, los alargamientos, los enunciados frustrados o truncados, "gestos, vacilaciones y otros ruidos", son "normales" en la comunicación oral, y "constituyen recursos importantes que usa el hablante para precisar el significado de una expresión, para multiplicar la emotividad de un enunciado, para generar espacios de participación con el interlocutor, o simplemente para respirar" (p. 7). Este trabajo, no obstante, constituye una intervención de tipo prescriptivista, al expresar que la presencia "reiterada y excesiva presencia" de tales fenómenos inciden positiva o negativamente en la "calidad del discurso" y al ofrecer una propuesta de evaluación de éste.

El trabajo de Jeong (2011), por su parte, ofrece una comparación de vacilaciones entre el español y el coreano. También retoma los tres tipos de vacilaciones propuestos por Laforest (1997) y expresa que se trata de elementos que intervienen "en los casos en que dos segmentos discursivos contiguos precisan establecer una relación entre sí" (p. 328), por lo que apoyan la continuidad global del discurso y "participan en la ilación de 
parcelas discursivas que guardan relaciones sintácticas y/o de significado más o menos estrechas" (Ibid.).

El interés del presente estudio se centra en la función y distribución que este tipo de fenómenos de la oralidad exhibe con respecto al grado de espontaneidad y planificación del discurso en una situación de habla formal que, como se verá, consta de secuencias discursivas bien delimitadas temporal y temáticamente. La situación de habla que se estudia son las entrevistas realizadas por legisladores de la Asamblea Legislativa del Distrito Federal ${ }^{3}$ (en adelante ALDF) a ciudadanos que compiten por obtener el cargo de ombudsperson, vacante en la Comisión de Derechos Humanos del Distrito Federal (en adelante $\mathrm{CDHDF}^{4}$ ), proceso que se llevó a cabo a finales de 2013.

Este trabajo reporta algunos hallazgos producto de una investigación de tipo etnográfico que permitió la conformación de un corpus a partir del cual se elaboraron análisis cuantitativos. Este acercamiento se realizó durante la VI Legislatura (2012-2015) de la ALDF, compuesta por 66 legisladores, 44 hombres y 22 mujeres. Esta institución legislativa organizó sus actividades en 37 comisiones legislativas (grupos de trabajo integrados por legisladores) abocadas cada una a agendas o temas de gobierno específicos, como transporte público, salud, presupuesto, educación, equidad de género, movilidad, entre otros. Las actividades de las comisiones legislativas representan una cara de la ALDF y una etapa dentro del proceso legislativo mexicano que, como ya se señaló, no ha sido explorado en estudios sociolingüísticos, pragmáticos y conversacionales.

De acuerdo con la Ley Orgánica de la ALDF (2008), las comisiones deben "reflejar la pluralidad de la Asamblea" para asegurar la participación de legisladores de todos los partidos políticos. Para establecer la muestra del estudio se preseleccionaron las comisiones que tuvieran mayor equilibrio de legisladores hombres y mujeres y de las 37 se eligió la de Derechos Humanos, pues del total de nueve legisladores que la formaron, cinco eran mujeres y cuatro hombres.

\footnotetext{
Órgano legislativo local de la capital mexicana, demarcación política que antes del 30 de enero de 2016 era reconocida como el "Distrito Federal" de México, y que posterior a esa fecha, y tras una serie de reformas realizadas desde hace alrededor de 15 años, se constituye como una entidad federativa -estado- que ha sido denominada "Ciudad de México". A partir de 2018, la ALDF cambió de nombre oficialmente a Congreso de la Ciudad de México.

4 "Organismo público autónomo [...] que tiene por objeto la protección, defensa, vigilancia, promoción, estudio, educación y difusión de los derechos humanos" (Ley de la Comisión de Derechos Humanos del Distrito Federal, Cap. 1, Art. 2.).
} 
En el marco de las atribuciones asignadas a la Comisión de Derechos Humanos de la ALDF por su Ley Orgánica, se encuentra la obligación de diseñar y ejecutar el proceso de búsqueda y selección del presidente honorario de la CDHDF (popularmente conocido como ombudsperson, o "defensor/a" de los derechos humanos de la ciudad). Fue este el evento particular en el que se centró esta investigación, la elección de un funcionario del gobierno de la Ciudad de México de entre un conjunto de aspirantes ciudadanos, sin adscripción oficial a algún partido político. La competencia por dicho cargo se dio mediante la presentación oralizada de una propuesta de trabajo ante los legisladores, quienes fueron los jueces encargados de elegir el perfil "idóneo" según sus criterios ${ }^{5}$. Es decir, la competencia por el cargo estuvo basada (además de la trayectoria profesional de los aspirantes), en la actuación discursiva-expositiva de su persona, de sus opiniones personales y de su propuesta de trabajo.

El estudio lingüístico de las vacilaciones en este entorno institucional propone un modelo híbrido que, como se verá más adelante, combina herramientas tanto del análisis de la conversación -en tanto la interacción, aunque altamente regulada, exhibe rasgos formales de la conversacióncomo de la sociolingüística variacionista - en tanto las reflexiones finales se realizan a partir de frecuencias o patrones cuantitativos. Estas frecuencias de ocurrencia o aparición en el corpus son correlacionadas con la unidad conversacional "secuencia".

Consideramos que una comprensión más completa de las funciones discursivas de los fenómenos identificados exige, en principio, el uso combinado de la teoría conversacionalista y de las metodologías cuantitativas asociadas con el variacionismo. En la elaboración del marco teórico hemos acudido a los estudios de la conversación de Sacks, Schegloff y Jefferson (1974), debido a que a pesar de tratarse de una interacción controlada en la construcción, administración y contenido de los turnos de habla, se presentan en ella una serie de condiciones propias de la conversación, según la perspectiva de estos autores: hay un cambio de interlocutores, y puede ser recurrente (p. 706); predominantemente una persona habla a la vez (Ibid.); el número de participantes es variable (p. 712); la conversación puede ser continua o discontinua cuando el tema se encadena, o no, en los turnos de otros participantes (p. 714); y aparecen técnicas para gestionar los turnos de habla (p. 716), que en este caso son todos autorizados por la diputada

\footnotetext{
5 La deliberación entre los legisladores sobre la elección del "mejor" aspirante para ocupar el cargo se dio en una reunión a puerta cerrada.
} 
presidenta de la Comisión legislativa, quien asigna los turnos de habla a los diputados en la secuencia de preguntas a los aspirantes, y autoriza a unos y otros el uso de la voz a lo largo del evento discursivo.

\section{EL FORMATO PARA LAS ENTREVISTAS APROBADO POR LOS LEGISLADORES}

Para llevar a cabo el proceso de selección de ombudsperson, los legisladores de la ALDF establecieron una serie de acuerdos y decisiones que quedaron asentados en las actas de las sesiones llevadas a cabo y que fueron publicados en la página web de la ALDF (www.aldf.gob.mx). En estas sesiones decidieron sobre las condiciones, reglas y formas que debían seguir los aspirantes que competían por el cargo, condiciones, reglas y formas que se encontraron en el nivel discursivo y que permitieron apreciar representaciones sociales hacia el uso de la lengua en este contexto, como lo fue, por ejemplo, el hecho de que la exposición estuviera delimitada temática y temporalmente.

El 9 de octubre de 2013, la Comisión legislativa comenzó a trabajar en el diseño del proceso para la elección de un nuevo titular, proceso que se dio a conocer públicamente en la $19^{\mathrm{a}}$ sesión ordinaria de la Comisión que tuvo lugar ese día; el orden del día abordó cinco asuntos:

1. Lectura y discusión y en su caso aprobación del acuerdo por el que se establece la ruta crítica ${ }^{6}$ del proceso de elección de la o el Presidente de la Comisión de Derechos Humanos del Distrito Federal.

2. Lectura, discusión y en su caso aprobación del acuerdo por el que se aprueba el contenido de la convocatoria para el proceso de elección de

6 La "ruta crítica" establecida fue la siguiente: 9 de octubre: publicación de la convocatoria (mismo día de la sesión); 11 al 17 de octubre: recepción de propuestas de candidatos; 20 de octubre: publicación de la lista de candidatos a ocupar el cargo; 21 al 28 de octubre: "recepción de opiniones" y notificación a los candidatos para la entrevista; 29 al 31 de octubre: realización de las entrevistas, en el orden en que los candidatos se registraron para el proceso; 1 de noviembre: "remisión a la Comisión de Gobierno para que emita su opinión"; 2 de noviembre: "sesión de trabajo de la Comisión para la discusión y en su caso aprobación del dictamen correspondiente" (Versión estenográfica de la 19 ${ }^{a}$ Sesión de la Comisión de Derechos Humanos de la ALDF, 9 de octubre de 2013). 
la o el Presidente de la Comisión de Derechos Humanos del Distrito Federal.

3. Lectura, discusión y en su caso aprobación del acuerdo por el que se aprueba el formato para el desarrollo de las entrevistas a las y los aspirantes a ocupar la Presidencia de la Comisión de Derechos Humanos del Distrito Federal.

4. Lectura, discusión y en su caso aprobación del acuerdo por el que se establece que se remitirá a la Comisión de Gobierno de esta H. Asamblea Legislativa las propuestas emitidas por las organizaciones, instituciones y entidades relacionadas en la materia, a efecto de que dicha Comisión señale su opinión.

5. Asuntos generales.

(Versión estenográfica de la $19^{a}$ Sesión de la Comisión de Derechos Humanos de la ALDF, 9 de octubre de 2013).

El punto de mayor interés para este trabajo es el tercero, correspondiente al acuerdo sobre el "formato para el desarrollo de las entrevistas". En la sesión, la diputada secretaria de la Comisión, Laura Iraís Ballesteros Mancilla, del Partido Acción Nacional (PAN), primera oposición en la VI Legislatura de la ALDF, dio lectura a dicho acuerdo sobre el formato:

1. Las entrevistas a las y los aspirantes se realizarán de forma individual ante el pleno de la Comisión de Derechos Humanos del Distrito Federal a partir del día 29 de octubre de 2013.

2. La Presidenta de la Comisión iniciará la entrevista dando la bienvenida a la o el aspirante a entrevistar.

3. Cada aspirante contará hasta con 15 minutos para realizar su exposición, la cual debe abarcar los siguientes aspectos:

a) Opinión personal respectiva al trabajo realizado hasta la fecha por la Comisión de Derechos Humanos del Distrito Federal.

b) Opinión personal respecto a perspectivas y retos de la Comisión de Derechos Humanos del Distrito Federal.

c) Propuesta de programa de trabajo.

4. Posteriormente habrá una ronda de preguntas donde harán uso de la palabra hasta por 3 minutos cada uno de los diputados integrantes de la Comisión de Derechos Humanos con la finalidad de realizar preguntas a la o el aspirante, quien contará hasta con 15 minutos para responder a todas las preguntas. Ninguna de las entrevistas podrá realizarse en forma de diálogo. 
5. Al final de la ronda de preguntas y respuestas cada aspirante podrá dar un mensaje final hasta por 5 minutos.

6. La Presidenta concluirá agradeciendo la asistencia a cada aspirante.

(Versión estenográfica de la $19^{a}$ Sesión de la Comisión de Derechos Humanos de la ALDF, 9 de octubre de 2013).

El formato para las entrevistas aprobado en la sesión se muestra esquematizado en la Tabla 1. Como ya se mencionó, este formato presenta una rígida delimitación temática de las secuencias del encuentro entre aspirantes y legisladores así como una delimitación temporal.

\begin{tabular}{|l|l|l|l|}
\hline & \multicolumn{1}{|c|}{ Secuencia } & \multicolumn{1}{|c|}{ Hablante/s } & Duración (aprox.) \\
\hline 1 & $\begin{array}{l}\text { Bienvenida y presentación del } \\
\text { aspirante. Lectura de currículum } \\
\text { vítae }\end{array}$ & Diputada presidenta & Menor a 5 minutos \\
\hline 2 & $\begin{array}{l}\text { Presentación de propuesta de } \\
\text { programa de trabajo }\end{array}$ & Aspirante & 15 minutos \\
\hline 3 & Formulación de preguntas al aspirante & Legisladores & $\begin{array}{l}\text { Variable (+/- 10 } \\
\text { min.) }\end{array}$ \\
\hline 4 & Respuesta a preguntas de diputados & Aspirante & 15 minutos \\
\hline 5 & Cierre & Aspirante & 5 minutos \\
\hline 6 & Agradecimiento y despedida & Diputada presidenta & Menor a 1 minuto \\
\hline
\end{tabular}

Tabla 1. Secuencias de las entrevistas

Esta rigidez de la entrevista está relacionada con la institucionalización del proceso de elección de ombudsperson, en el que los legisladores pretendieron construir un modelo que ofreciera las mismas oportunidades y entornos de participación a los aspirantes al cargo; detrás de esta intención parecería asumirse que el alto control de la interacción y la administración temática y temporal de sus secuencias discursivas son acciones que garantizan "condiciones de igualdad" a los participantes.

Resulta importante cuestionar y reconocer las limitaciones de este supuesto de "participación igualitaria", por dos razones: La primera es que, aunque esta estandarización discursiva de concursos a cargos ofrecidos por instituciones de gobierno constituye una práctica común en los sistemas democráticos modernos, no significa necesariamente que su acatamiento garantice un proceso "justo" y "transparente", pues prácticas de corrupción podrían operar en distintas formas y niveles. La segunda es que este acto de "codificación interactiva" parece asumir y esperar que los estilos discursivos 
individuales de los participantes en el proceso son, o deberían ser, todos iguales, y por lo tanto, la aprobación de un formato como éste constituye una expresión de normatividad lingüística y paralingüística hacia lo que los legisladores valoran como el candidato o la candidata "ideal" para ser ombudsperson, que constriñe, uniforma y ajusta el uso de la lengua a este entorno específico de participación, contribuyendo a crear un registro de habla protocolar, altamente planificado y con gran atención a la forma ${ }^{7}$.

Las secuencias en la Tabla 1 aparecen numeradas según el orden cronológico de la interacción. A pesar de que el punto 4 del formato aprobado establece que "Ninguna de las entrevistas podrá realizarse en forma de diálogo", este sí existió pues se presenta una alternancia de turnos (Sacks et al. 1974), así como una "sincronización interaccional", que de acuerdo con Kerbrat-Orecchioni aparece cuando dos interlocutores están comprometidos en el intercambio y estos producen señales de engagement mutuel (1996, Cf. p. 4-5), con la particularidad de que la alternancia de turnos está regulada por la diputada presidenta, quien ocupa la función de administradora de turnos (Ibid, p. 30). Es posible, además, apreciar un "par adyacente" de tipo pregunta-respuesta ( 3 y 4 en Tabla 1), a pesar de que 3 se constituye como una multiplicidad de voces que se erigen como representantes de la institución; esta multiplicidad puede observarse como un solo interlocutor: la naturaleza discursiva de 3 configura así un emisor (el "bloque" de voces compuesto por los legisladores), y un destinatario en 4 (el o la aspirante a quien se dirigen las preguntas).

7 Un sustento empírico a esta observación se encontró en el caso de Irina Layevska Echeverría, ciudadana que compitió por el puesto, que muestra que en el diseño del formato de entrevista de los legisladores se aprecian lugares comunes sobre la práctica democrática, así como representaciones sobre la igualdad que no coinciden con la compleja realidad social. En este caso, Echeverría presentó una propuesta que no cumplió con las expectativas discursivas de los legisladores ni los requerimientos temporales de las secuencias que estos establecieron. Además, Echeverría no escribió las preguntas que los legisladores hicieron "en bloque" debido a una discapacidad motriz, por lo tanto dio respuesta solo a aquellas que recordó cuando llegó el momento de responderlas. Casos como la participación de Echeverría en este proceso representan un reto metodológico a la investigación, así como un reto a la validez de las regularidades del comportamiento verbal establecidas exclusivamente a partir de métodos cuantitativos. La entrevista a Irina Layevska no se tomó en cuenta en este estudio debido a que la frecuencia de vacilaciones presentó patrones muy distintos a los demás hablantes; la amplia diferencia numérica parece estar relacionada con factores fisiológicos que, como en cualquier hablante, contribuyen a formar el estilo discursivo individual. Estos factores fisiológicos, no obstante, parecen constituir una variable de mayor peso que la situación social o la naturaleza de las secuencias, en el caso de Echeverría, algo que no ocurre en el resto de los participantes. Este caso en particular plantea la necesidad de problematizar lo que significa "participar en igualdad de condiciones". 


\section{VACILACIONES Y FLUIDEZ ORAL}

No es posible ofrecer una definición de "vacilación" sin acudir al carácter prescriptivo de la gramática oracional, del discurso impreso y de una cultura sobre la "fluidez oral", como conjunto de ideas naturalizadas en la sociedad, que se han forjado históricamente desde la Grecia antigua, y que ha sido alimentada por lo que Walter Ong denomina, acudiendo a Haugen, "grafolecto": una lengua formada por una profunda dedicación a la escritura (1987, p. 17). Esta cultura sobre la fluidez oral se encuentra fuertemente arraigada y ha provocado, en opinión de Ong, que la lengua escrita haya quedado desprovista de la posibilidad de ser imaginada como lengua en voz alta, es decir, como lengua oral, además de que ha alimentado, especialmente en sociedades de profunda tradición literaria, el deseo de acercar la lengua oral a esa gramática oracional, a ese discurso y a esa lengua impresos. No obstante, los enunciados de la lengua en uso, como sabemos, no son reproducciones de la lengua escrita.

Postular una definición de "vacilación" implica, por lo tanto, reconocer que existen producciones orales que intervienen en la forma de la oración y que no son reconocidas o tomadas en cuenta como elementos que pueden participar en un análisis gramatical. Hablar de las "vacilaciones" como objeto de estudio, desde las ciencias del lenguaje, como desde el conocimiento común, demanda acudir, de una forma u otra, a una idealización del discurso y de la lengua que se encuentra atravesada por la escritura. Partiendo de reconocer esa relación entre la noción de vacilación-oración/lengua escrita, las vacilaciones son vistas como aquellos elementos considerados 'extraños', 'intrusos' o 'ajenos' a la oración como unidad de análisis y comunicación. Estos elementos constituyen autointerrupciones del hablante dentro del propio enunciado o enunciados que componen su turno de habla, realizadas mediante elementos léxicos, no léxicos o silencios, en apariencia "innecesarios" desde un punto de vista prescriptivo. Estas autointerrupciones son valoradas socialmente como indeseables por un conjunto de ideas cuyo origen podría rastrearse desde la tradición retórica occidental del siglo $\mathrm{V}$ a. C. ( $C f$. López Eire 2007). Algunas expresiones contemporáneas de este conjunto de ideas se aprecian en manuales sobre el "bien hablar" y particularmente sobre el "bien hablar en público" ( $C f$. Instituto Cervantes 2008) $)^{8}$. Las vacilaciones

\footnotetext{
8 Para una construcción discursiva de la 'fluidez oral' con perspectiva histórica, ver Quesada, 2017.
} 
aparecen en dos posibles posiciones: 1) al interior de las unidades sintácticosemánticas, ya sean frases, cláusulas u oraciones, fragmentando su unidad, o 2) en las fronteras que enmarcan estas unidades. No entran en esta definición eventos como el hipo, estornudar o eructar, ni aquellas autointerrupciones que se explican porque el entorno físico ha ocasionado una alteración del discurso, como, por ejemplo, la sirena de una ambulancia que pasa por la calle.

De acuerdo con varios autores (Clark 1994; Duez 2001), una de las características más representativas de las vacilaciones es que tienden a ser más frecuentes en situaciones de habla espontánea y menos frecuentes en discursos planificados de tipo formal. Sin embargo, estas condiciones no son las únicas posibles para entender la frecuencia y distribución de estos fenómenos, pues otros factores de tipo cognitivo, psicológico y social podrían también operar como variables que determinan su "comportamiento".

Como se mencionó, las vacilaciones forman parte de un conjunto de ideas sobre la fluidez oral que puede encontrarse en manuales o guías sobre el "bien hablar"; en estos materiales son comunes las nociones de "torpe", "tonto", "inarticulado", "no preparado", asociadas a quien vacila al hablar, mientras que la persona que vacila menos es percibida como "inteligente", "segura" o "buena oradora". Este conjunto de ideas es un producto histórico que sigue siendo reforzado desde los diversos productos de la industria cultural (consumo mediático, entretenimiento), las instituciones de gobierno y los procesos de estandarización escolar. La valoración positiva hacia una presentación oral pública, por ejemplo, no depende exclusivamente de la relevancia y organización del contenido, depende también de la manera en que dicha presentación ha sido actuada, "performada", y de si esa actuación coincide con las expectativas y modelos que el auditorio tiene por "buena" o "mala", "correcta" o "incorrecta", "inapropiada" o "efectiva", es decir, si esa actuación coincide con el conjunto de ideas sobre el "buen orador", el "buen expositor" o el "buen funcionario" depositadas en el auditorio. Aunque no se trata de valoraciones enunciadas de manera explícita, un auditorio por lo común espera que una presentación oral se desarrolle libre de vacilaciones, y su menor o mayor ocurrencia incidirá en la forma en la que dicho discurso será valorado. 


\subsection{TIPOS DE VACILACIONES}

Con la definición de vacilación antes expuesta se procedió a transcribir y etiquetar el corpus, compuesto por una muestra de 9 entrevistas $^{9}$ del total de 29 que se realizaron en el proceso de elección de ombudsperson de la CDHDF. La transcripción fue de tipo ortográfica, complementada con algunas convenciones del sistema de etiquetado del Proyecto para el Estudio Sociolingüístico del Español de España y América (PRESEEA). Dicho sistema cuenta con la etiqueta " $<$ vacilación/ $>$ ", definida como un "titubeo breve", usada para "marcar pequeños titubeos que no constituyen palabras". Para los propósitos descriptivos de la investigación, esta definición resultó insuficiente ante la diversidad de fenómenos de vacilación que se encontraron en el corpus. Así, se crearon las siguientes etiquetas, registradas en el corpus:

- Sonido breve $\mathrm{e}^{10}:<$ sonido $>$

(1) $\underline{e}\langle$ sonido $>$ yo en lo particular considero que cuando un derecho

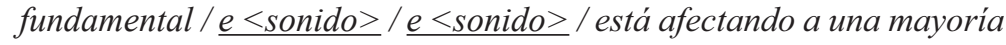

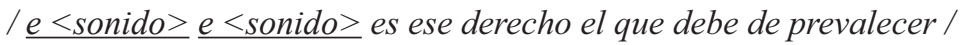

- Repetición no intencional: <repetición>

(2) / agradecemos la presencia del /// del <repetición> diputado Gaviño / quien nos honra con su presencia / en esta Comisión /

- Frases cortadas: <frase_cortada $>$

(3) / la $<$ frase cortada $>/<$ reparación $>$ el respeto a la libre manifestación pero al mismo tiempo al libre tránsito /

- Palabra cortada: <palabra_cortada $>$

(4) / la corresponsabilidad de la Comisión de Derechos Humanos / en la ciu- <palabra_cortada $>$ / <reparación > hacia la ciudad /

- Alargamiento, de sonido o de palabra: $<(:)>$

(5) / $\underline{e}\langle(:)\rangle /$ y qué estrategias implementaría para la violación de derechos humanos en el Distrito Federal /

\footnotetext{
Incluyen un total de 22 participantes; 8 horas de videograbación.

En oposición a sonido largo, y que puede ser vocálico o consonántico.
} 
(6) / pero $<(:)>$ a diario se dan / en estos casi nueve millones de habitantes /violaciones a derechos humanos en materia administrativa /

En los ejemplos (3) y (4) aparece la etiqueta " $<$ reparación>” la cual indica que a continuación sigue una autocorrección al corte de palabra o frase que produjo el hablante. Aunque no se incluye aquí un análisis sobre la reparación como tal, su etiquetado es importante pues permite apreciar la posible causa de esos cortes (el material que ha sido descartado y que es el objeto del corte). La reparación no es considerada una vacilación en tanto no implica por sí misma una ruptura de la cadena hablada, sino que precisamente permite la continuación de ésta, y se presenta por lo tanto como una "solución" a dicha ruptura ${ }^{11}$.

A propósito de la imposibilidad de emplear las etiquetas con las que cuenta actualmente el sistema PRESEEA, es necesario subrayar que éste consiste en una propuesta de "marcas y etiquetas mínimas y obligatorias" que obedece unos objetivos comunes y unos criterios generales, entre los que se encuentran, entre otros, el evitar "las redundancias y los esfuerzos desproporcionados", el eliminar "el componente interpretativo", además de no tener como finalidad el señalamiento de "posibles objetos de estudio". El presente manuscrito busca justamente visibilizar la riqueza detrás de los fenómenos de vacilación mediante una labor de identificación y descripción más precisa de los mismos, así como evidenciar que los esfuerzos en su transcripción, por poco económica que pueda parecer, pueden aportar a la comprensión del proceso de elaboración discursiva.

\section{EL CORPUS Y LOS DATOS}

Luego de transcribir y etiquetar las 9 entrevistas en un procesador de textos, se creó una base de datos (hoja de cálculo) en la que se registró una entrada por cada enunciado; estas entradas son similares a las de los ejemplos (1) a (6) antes presentados y subsiguientes. Para la segmentación en enunciados se buscó que cada uno tuviera cierta independencia sintáctico-semántica,

11 La reparación ofrece un rico fenómeno para una profundización posterior; se trata de un momento de la enunciación particularmente sensible al contexto de habla, es decir, representa un indicio del automonitoreo discursivo. 
así como una intención comunicativa orientada hacia el tema general de la exposición del hablante, como hacia la secuencia discursiva de que se tratara; es decir, la segmentación procuró identificar unidades comprensibles según el tipo de discurso y la función de dicha unidad en relación con este.

Cada enunciado fue clasificado de acuerdo a los seis fenómenos de vacilación que se presentaron en $\$ 3.1$ y que en conjunto constituyen la variable dependiente (las vacilaciones); sus variantes son: sonido breve, repetición, sonido largo, alargamiento de palabra, palabra cortada y frase cortada. Las variables independientes que se consideraron para la elaboración de la hoja de cálculo fueron las siguientes ${ }^{12}$ :

a) Secuencia (Lectura de Currículum / Presentación / Preguntas / Respuestas / Cierre / Monitoreo13)

b) Rol del hablante que produjo el enunciado (aspirante / legislador)

c) Sexo del hablante que produjo el enunciado (hombre / mujer)

d) Realización de reparación posterior al fenómeno de vacilación (sí / no) 14

e) Repetición del fenómeno de vacilación ${ }^{15}$ (sí / no).

La captura de los enunciados en la hoja de cálculo dio un total de 2,575. Su caracterización de acuerdo con las variables permitió organizar el corpus para efectuar el análisis que aquí se presenta. La distribución total de fenómenos de vacilación por tipos, ordenados de mayor a menor, aparece en la Tabla 2.

\begin{tabular}{|l|l|l|}
\hline Fenómeno & Número de ocurrencias & Porcentaje \\
\hline Sonido breve & 579 & 22.48 \\
\hline Repetición & 566 & 21.98 \\
\hline Frase cortada & 494 & 19.18 \\
\hline Sonido largo & 451 & 17.51 \\
\hline
\end{tabular}

12 Entre paréntesis aparecen las variantes de cada una.

13 Se trata de intervenciones protocolares, en su mayoría a cargo de la diputada presidenta, como la apertura de sesión y la lectura del orden del día; autorizaciones para la transición de secuencia o de turnos; llamados al orden en la sala, llamados a que el candidato se ajuste a los tiempos establecidos; solicitudes de aspirantes a que se le repita alguna pregunta, entre otros casos, cuya suma corresponde a un tipo discursivo sumamente diverso.

14 Esta variable corresponde a las palabras y frases cortadas, casi en su totalidad.

15 Esta variable no se tomó en cuenta, dado que, en tanto constituye una repetición, el fenómeno quedó contabilizado en la variante 'Repetición' de la variable dependiente. 


\begin{tabular}{|l|l|l|}
\hline Alargamiento de palabra & 251 & 9.74 \\
\hline Palabra cortada & 234 & 9.08 \\
\hline TOTAL & 2575 & 100.0 \\
\hline
\end{tabular}

Tabla 2. Número de vacilaciones por tipo en el corpus

Existen muchas posibilidades analíticas de los datos de la Tabla 2 a partir de las variables independientes ya mencionadas. En este estudio, no obstante, se aborda exclusivamente el análisis que relaciona las cifras de la Tabla 2 (la variable dependiente - las vacilaciones) con la variable independiente 'Secuencia' (inciso a), en la que se ubica el enunciado (Lectura de Currículum, Presentación, Preguntas, Respuestas, Cierre, Monitoreo). Este cruce de variables permite ofrecer explicaciones a las frecuencias de ocurrencia en función de la estructura conversacional del evento y los distintos grados de planificación discursiva que presenta.

\section{VACILACIONES POR SECUENCIA Y PLANIFICACIÓN DISCURSIVA}

Como se expuso en $\S 2$, el formato de las entrevistas realizadas por la Comisión de Derechos Humanos de la ALDF para elegir al ombudsperson de la CDHDF surgió de una propuesta creada por los legisladores. En dicho formato el diálogo "no se permitió", de modo que las transiciones de turno y de secuencia estuvieron estrictamente delimitadas en tiempo y controladas por la diputada presidenta, única voz autorizada para regular estas transiciones. En la Tabla 3 aparecen los fenómenos de vacilación más frecuentes según la secuencia de la entrevista.

\begin{tabular}{|l|l|l|l|l|l|}
\hline & $\begin{array}{l}\text { Lectura } \\
\text { de CV }\end{array}$ & Presentación & Preguntas & Respuestas & Cierre \\
\hline 1 & Frase cortada & Frase cortada & Sonido breve & Repetición & Repetición \\
\hline 2 & $\begin{array}{l}\text { Palabra } \\
\text { cortada }\end{array}$ & Repetición & Sonido largo & Sonido breve & Frase cortada \\
\hline
\end{tabular}




\begin{tabular}{|c|c|c|c|c|c|}
\hline \multirow[t]{2}{*}{3} & \multirow[t]{2}{*}{ Sonido largo } & \multirow[t]{2}{*}{ Sonido breve } & \multirow{2}{*}{$\begin{array}{l}\text { Alargamiento } \\
\text { de palabra }\end{array}$} & \multirow[t]{2}{*}{ Frase cortada } & Sonido breve \\
\hline & & & & & $\begin{array}{l}\text { Palabra } \\
\text { cortada }\end{array}$ \\
\hline 4 & Repetición & $\begin{array}{l}\text { Palabra } \\
\text { cortada }\end{array}$ & Repetición & Sonido largo & Sonido largo \\
\hline \multirow[t]{2}{*}{5} & \multirow[t]{2}{*}{ Sonido breve } & \multirow[t]{2}{*}{ Sonido largo } & \multirow[t]{2}{*}{ Frase cortada } & $\begin{array}{l}\text { Alarg. de } \\
\text { palabra }\end{array}$ & \multirow[t]{2}{*}{$\begin{array}{l}\text { Alargamiento } \\
\text { de palabra }\end{array}$} \\
\hline & & & & $\begin{array}{l}\text { Palabra } \\
\text { cortada }\end{array}$ & \\
\hline 6 & $\begin{array}{l}\text { Alargamiento } \\
\text { de palabra }\end{array}$ & $\begin{array}{l}\text { Alargamiento } \\
\text { de palabra }\end{array}$ & $\begin{array}{l}\text { Palabra } \\
\text { cortada }\end{array}$ & & \\
\hline
\end{tabular}

Tabla 3. Frecuencia de vacilaciones por secuencia en la interacción

La Tabla 3 permite apreciar un "movimiento" de tipos de vacilación según el desarrollo temporal del evento (plano horizontal en tabla). Para entender esta variación en la distribución de fenómenos se toma en cuenta el grado de planificación discursiva que cada secuencia presenta.

Por elaboración discursiva oral se entiende aquí el proceso que involucra elegir de entre los recursos léxicos, prosódicos, sintácticos y semióticos en general con los que el hablante construye un mensaje, así como de los elementos paralingüísticos que lo acompañan en la complementación de significados (tono de la voz, velocidad de elocución, expresión facial, gesticulación, entre otros), aquellos elementos según evalúe adecuados según la interacción o situación comunicativa en la que se encuentra. Esta elaboración discursiva puede ser 1) espontánea (no planificada), en la que el proceso de planear/pensar y el acto de hablar se presentan de modo casi simultáneo, o 2) planificada, donde el hablante ha preparado su discurso con anticipación a la situación interactiva, quizás lo ha practicado e incluso memorizado. En este segundo tipo de elaboración discursiva el hablante ha contado con tiempo por anticipado, lapso de tiempo que aleja el proceso de planear/pensar del acto de hablar.

Las expresiones de la lengua escrita, como las de la lengua oral presentan grados de planificación y espontaneidad, rasgos que han sido integrados en algunos modelos para el análisis de la lengua (Briz 1998; Koch y Oesterreicher 1990 [2007]). Es necesario tomar en cuenta que por muy espontáneo que sea el acto de escribir, requiere mayor tiempo que hablar, quien escribe cuenta por lo general con mayores oportunidades para planificar el mensaje previas a su materialización gráfica. La lengua escrita ofrece así más oportunidades que la lengua oral y su inmediatez para el ajuste, edición o "mejoramiento" del mensaje antes de que éste sea entregado al destinatario. 
De igual manera, por altamente planificada que sea la lengua oral, no deja de ser una práctica en la que hablar y pensar suceden casi al mismo tiempo, y donde el hablante realiza un automonitoreo del discurso ( $C f$. Levelt 1983) en el que las condiciones externas de uso de la lengua, es decir, el contexto social o interpersonal, motivarían un mayor o menor "proceso editorial" cuya "evidencia material" serían fenómenos como las rupturas sintácticas, las vacilaciones, los sonidos, las repeticiones no intencionales, los refraseos, las reparaciones y reformulaciones, fenómenos que en conjunto pueden ser referidos como marcas de una "escasa destreza oratoria" (Instituto Cervantes 2008, p. 113), un atentado contra la fluidez oral como ideal y contra la estética verbal promovida por la tradición retórica occidental.

A partir de estas observaciones, es posible identificar que las secuencias de la Tabla 3 muestran distintos grados de planificación discursiva, es decir, incluyen discursos que han sido unos más planificados que otros, y discursos que reflejan mayor o menor grado de espontaneidad.

\subsection{GRADO DE PLANIFICACIÓN DISCURSIVA}

De los planteamientos anteriores se sigue que las secuencias que se muestran en el eje horizontal de la Tabla 3 (Lectura de CV, Presentación, Preguntas, Respuestas y Cierre) presentan distintos grados de planificación discursiva. En algunas de ellas se da la oralización de un texto escrito exclusivamente (Lectura de CV), en otras la alternancia entre lectura y enunciación espontánea (Presentación), en otras la espontaneidad simulada o planificada (Cierre) y en otras la elaboración discursiva espontánea (Respuestas). A partir de estas observaciones, las secuencias de las entrevistas se agruparon en tres posibles grados de planificación: 1) discurso planificado; 2) discurso semiplanificado y 3 ) discurso no planificado ${ }^{16}$.

Las secuencias Lectura de CV, Presentación y Cierre se consideraron las más planificadas; la Lectura de $\mathrm{CV}$, a cargo de la diputada presidenta, es planificada, pues se trató de la oralización de un texto escrito: el paso del medio gráfico al medio fónico de un documento tan planificado como lo es una hoja de vida. La Presentación y el Cierre, ejecutadas por los aspirantes al cargo también se consideraron momentos altamente planificados en función

\footnotetext{
16 Por supuesto esta categorización no es exhaustiva, pues cada secuencia puede mostrar estos y otros grados de planificación discursiva, que además alternan constantemente al interior de una u otra secuencia; sin embargo, permitió encontrar patrones para explicar la ocurrencia de vacilaciones en el corpus.
} 
de que, como aparece en los acuerdos 3 y 5 del "formato para el desarrollo para las entrevistas" (v. §2), se solicitó preparar una opinión, una propuesta de trabajo y un cierre sobre el tema de los derechos humanos en la Ciudad de México y sobre el trabajo de la CDHDF, para lo que contaron con 20 días de anticipación (el acuerdo se aprobó el 9 de octubre de 2013 y las entrevistas se realizaron entre el 29 y el 31 del mismo mes).

La secuencia de Preguntas a cargo de los legisladores se consideró semiplanificada, pues estuvieron en su mayoría motivadas por las presentaciones de los aspirantes. Hubo preguntas sobre su trayectoria profesional o inquietudes específicas que algunos legisladores plantearon a todos los aspirantes, independientemente del material expuesto por estos en la Presentación. En cualquier caso, los legisladores contaron con tiempo para elaborar las preguntas, que pudo ser el lapso de quince minutos que duró la primera parte de la entrevista, es decir, durante la Lectura de CV y la Presentación.

Finalmente, la secuencia de Respuestas se consideró no planificada. Aunque los aspirantes pudieron imaginar o anticipar lo que los legisladores les preguntarían, no lo tenían por seguro, y por lo tanto se asume que no contaron con un plan discursivo estructurado previamente para responder. En la Figura 1 se presenta la distribución de las vacilaciones por tipo, según el grado de planificación en el que se han agrupado las secuencias.

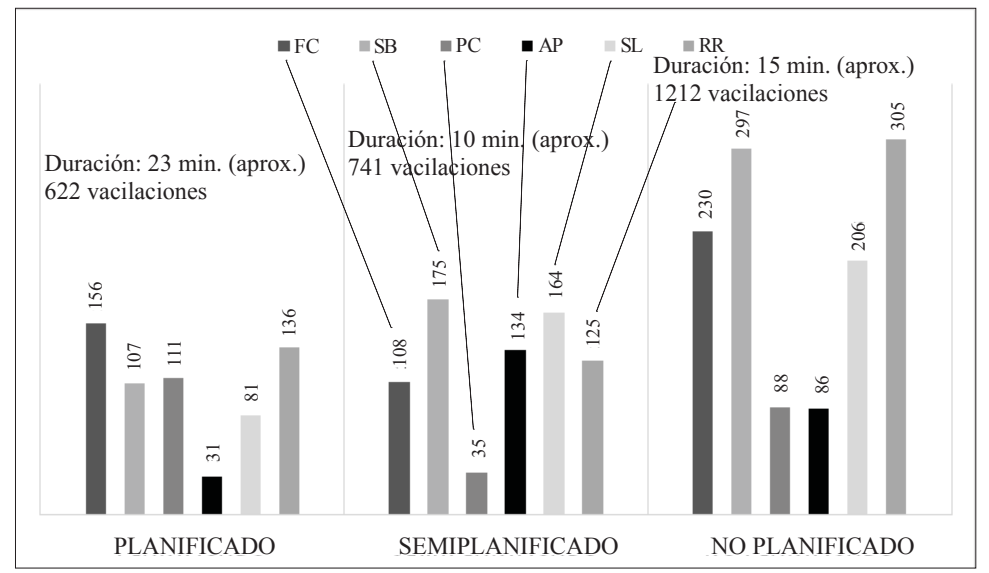

Figura 1. Vacilaciones por grado de planificación del discurso ${ }^{17}$

$17 \quad \mathrm{FC}=$ Frase cortada $; \mathrm{SB}=$ Sonido breve $; \mathrm{PC}=$ Palabra cortada $; \mathrm{AP}=$ Alargamiento de palabra; $\mathrm{SL}=$ Sonido largo; $\mathrm{RR}=$ Repetición. 
El primer dato que salta a la vista en la Figura 1 es la variación en el total de vacilaciones que aparecen por grupo, especialmente el hecho de no ser proporcional a la duración total aproximada de las secuencias agrupadas; esto contribuye a confirmar que la mayor o menor aparición de vacilaciones está relacionada directamente con el tipo de discurso según el grado de planificación en que se presentan: el segmento de mayor duración (23 minutos), correspondiente a discurso planificado, es precisamente el que menor número de fenómenos presenta, mientras que el segmento de discurso no planificado casi duplica esa cifra a pesar de que dura aproximadamente 8 minutos menos.

De los datos de la Figura 1 se extrae que la suma de palabras y frases cortadas, como fenómenos hasta cierto punto similares por el hecho de implicar un corte en la cadena hablada para (en la mayoría de los casos) introducir una reparación, constituyen fenómenos proporcionalmente más frecuentes en secuencias planificadas que en la suma correspondiente a segmentos semiplanificado y no planificado. Esta suma de FC y PC representa $43 \%$ del total de vacilaciones en discurso planificado, $19.3 \%$ en discurso semiplanificado y $26.2 \%$ en discurso no planificado. Estas cifras podrían indicar que en discursos planificados como la Presentación y el Cierre, e incluso la Lectura del CV, el tipo de discurso provocaría que el hablante mantenga el plan que ha elaborado con antelación, como si se tratase de un esquema mental al cual debe apegarse; el esfuerzo cognitivo y creativo que ha implicado esa planificación anticipada podrían motivar una fidelidad hacia ese plan, que en consecuencia crearía una mayor tendencia hacia el automonitoreo, reflejado en la mayor aparición de palabras y frases cortadas ${ }^{18}$.

No obstante, por mucho que el discurso haya sido planificado, ensayado o memorizado, este debe enfrentar la realidad social; ese plan discursivo, al trasladarse a esa realidad atraviesa por adaptaciones necesarias para dar respuesta a las diversas situaciones de la interacción. En (7), (8), (9) y (10) se presentan algunos ejemplos de palabras y frases cortadas extraídas de secuencias discursivas planificadas:

(7) / número uno / es necesario tomar medidas para abe- $<$ palabra_cortada $>$ $<$ reparación $>$ abatir la sobrecarga de trabajo / en las visitadurías generales

18 Esta observación se refuerza al considerar que el 91.8\% de todas las frases y palabras cortadas en el corpus vienen acompañadas de una reparación, y esta reparación parece ser el motivo para realizalas. 
(8) / me provo- <palabra cortada $><$ reparación $>$ me propongo realizar jornadas de trabajo / con cada una de las áreas de gobierno /

(9) / la convocatoria emitida para la Asamblea <frase cortada > / $<$ reparación> por la Asamblea del Distrito Federal / para elegir al ombudsperson /

(10) / la gran aportación de la reforma en derechos humanos fue devolver / el sartén <frase cortada $>/<$ reparación > el mango del sartén / a la sociedad/

En la Figura 1, la suma de los fenómenos sonidos breves y repeticiones, sumamente extendidos en toda la interacción (representan el $44 \%$ del total de vacilaciones encontradas en toda la muestra), son los casos más comunes en las secciones de discurso semiplanificado y no planificado, aunque no en las secciones de tipo planificado. En la mayoría de los casos el material repetido corresponde a palabras de la "clase cerrada" (determinantes, pronombres, preposiciones y conjunciones, principalmente) (Schachter y Shopen 2007, p. 23), que por lo general tienen longitud de una sílaba. Este valor silábico puede equipararse en duración con los sonidos breves, que casi en su totalidad son vocálicos. Es por esta razón que se propone agruparlos. La suma de repeticiones y sonidos breves para el primer segmento (planificado) representa 39\%; para el segundo (semiplanificado) $40.4 \%$ y para el tercero (no planificado) $49.6 \%$. Se aprecia entonces que, aunque son marcas de uso extendido en cualquiera de los tres tipos de discurso, lo son más en los discursos no planificados donde representan prácticamente la mitad del total de vacilaciones.

Los ejemplos (11), (12), (13) y (14) incluyen casos de repeticiones y sonidos breves en discursos no planificados:

(11) / porque además / no es sólo responsabilidad / del / del <repetición $\rangle$ Distrito Federal /

(12) / o sea es una cuestión de lógica / simplemente si no hay ninguna medida para / para <repetición $>$ para <repetición $>$ a $<$ sonido $>/ e<(:)>/$ agarrar ese problema / pues / obviamente lo vamos a seguir repitiendo /

(13) / el Tribunal Europeo de Derechos Humanos / en bajo / cuyajurisdicción / e $<$ sonido $>$ conviven e $<$ sonido $>$ / también estados que tienen / una una $<$ repetición $>$ legislación más cerrada /

(14) /ya después platicaremos de esos temas que son/realmente importantes para / para <repetición $>$ la ciudad e $<$ sonido $>$ / y no sólo para el Poder Judicial / 
Los sonidos largos y alargamientos de palabra también pueden agruparse en tanto consisten en la prolongación de un sonido generalmente vocálico, que recae sobre el segmento final de un elemento léxico, o sobre un elemento no léxico que se alarga y se inserta tanto en los límites como al interior de frases, cláusulas y oraciones ${ }^{19}$. En la Figura 1 la suma de estos dos fenómenos para discursos planificados representa solo $18 \%$, mientras que alcanza $40.2 \%$ para discursos semiplanificados y $24.1 \%$ para no planificados.

Ejemplos de sonidos largos y alargamientos de palabra en discursos semiplanificados aparecen en los ejemplos (15), (16), (17) y (18):

(15) / número dos / e $<(:)>$ y es otra pregunta también que las / o ese ces ${ }^{20}$ nos han hecho llegar /

(16) / e $\langle$ (:) $\rangle$ para nosotros es un privilegio contar con alguien con una formación académica tan sólida /

(17) / sino también la cuestión de los servicios / e <sonido $>$ es la discrepancia entre la $\langle(:)\rangle<$ frase cortada $>/<$ reparación $>$ el número

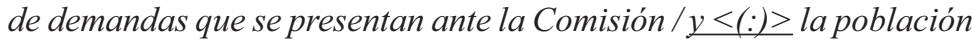
existente en el Distrito Federal /

(18) /// y también e <sonido > le preguntaría e $<(:)>$ // cuál cree usted que $\leq(:)\rangle<$ frase_cortada $>/<$ reparación $>$ qué está haciendo falta en la Comisión del Distrito Federal / en atención a las víctimas //I

\section{FUNCIONES DE LAS VACILACIONES}

Como se ha visto, la gráfica de la Figura 1 organiza los fenómenos de vacilación por secuencias de la interacción, a partir del grado de planificación observable en cada una de ellas (discurso planificado, semiplanificado y no planificado). Con la intención de presentar una propuesta que permita entender la función discursiva de las vacilaciones se retoman exclusivamente los segmentos de discurso planificado y discurso no planificado, para así

\footnotetext{
19 La profundización en el estudio de la posición de este y otros fenómenos de vacilación en este y otros corpus resultaría de gran valor desde una perspectiva sintáctica. Por ahora dicha perspectiva rebasa los propósitos del trabajo.

20 Se refiere a las siglas OSC, "organizaciones de la sociedad civil".
} 
contrastar con mayor claridad la variación de estos fenómenos en atención a la hipótesis de planificación. Para esto, las secuencias identificadas como de discurso semiplanificado han sido integradas a las de discurso no planificado.

Al hacer esta reagrupación, la proporción de vacilaciones es mucho mayor en el conjunto de secuencias no planificadas y semiplanificadas (Preguntas, Respuestas y Monitoreo), que en las planificadas (Lectura de currículum, Presentación y Cierre): la suma de vacilaciones en las secuencias no planificadas y semiplanificadas representa el $75.8 \%$ del total de la muestra. Esto significa en términos generales que las vacilaciones son más empleadas al producir discursos que poseen mayores rasgos de espontaneidad, es decir, aquellos que no cuentan con un plan discursivo previo. En orden de mayor a menor, el porcentaje de los fenómenos en las secuencias no planificadas (incluyendo semiplanificadas), son:

1) Sonido breve:

$24.2 \%$

2) Repetición:

$21.9 \%$

3) Sonido largo:

$18.8 \%$

4) Frase cortada:

$17.3 \%$

5) Alargamiento de palabra:

$11.2 \%$

6) Palabra cortada:

$6.4 \%$

Como se vio en el apartado anterior, estos seis fenómenos se agrupan según se "comportan" en el tipo de discurso. De tal manera, para las repeticiones y los sonidos breves se observa que funcionan en el corpus como pausas oralizadas de conexión (POC). Se retoma el término "pausa oralizada" empleado por varios autores (Duez 2001b; Jeong 2011) pues el hablante la realiza aparentemente para evitar una pausa de silencio. Esos milisegundos, más que constituirse como estrategias que le dan tiempo a elaborar sus enunciados, le apoyan a restringir vacíos en el flujo oral. Estos dos fenómenos podrían reflejar por lo tanto un impulso del hablante por saturar de sonido el espacio de tiempo que le ha sido otorgado en un contexto como éste.

Los sonidos largos y los alargamientos de palabra, por su parte, sí se consideran una estrategia o un apoyo de los hablantes para ganar tiempo en la planificación discursiva y por lo tanto su función sería de planificadores discursivos (PD). Una evidencia cuantitativa que refuerza esta propuesta es el hecho de que el $84 \%$ de estos fenómenos combinados ocurrieron dentro de secuencias que no son planificadas y el $16 \%$ restante en planificadas.

Finalmente, las palabras y frases cortadas pueden clasificarse también como fenómenos similares, puesto que ambos implican un corte del flujo oral realizado por un silencio, elemento léxico, sonido breve o largo $(C f$. "editing term", en Levelt 1983, p. 70), y porque en la mayoría de los casos 
(91.8\%) ocurren para realizar una reparación o reformulación discursiva. Es decir, las palabras y frases cortadas comparten la característica de aparecer debido a que en este contexto comunicativo el hablante intenta producir un mensaje claro, preciso y sin ambigüedades, lo que motiva una mayor aparición de autocorrecciones, las cuales demandan cortes de palabras y frases más frecuentes. Se propone la denominación mecanismos de edición (ME) para estos dos fenómenos, retomando la terminología de Levelt sobre el automonitoreo (op. cit.). Los ME podrían ser una evidencia material (oral) o reflejo del proceso de elaboración discursiva no planificada, cuando toman lugar los fenómenos de tipo PD.

Esta propuesta persigue representar las funciones de las vacilaciones según aparecen en el corpus. Su distribución porcentual en toda la muestra aparece en la Tabla 4.

\begin{tabular}{|l|c|c|c|c|}
\hline & POC & PD & ME & TOTAL \\
\hline Planificado & $39.0 \%$ & $18.3 \%$ & $42.7 \%$ & $100 \%$ \\
\hline No planificado $^{21}$ & $46.1 \%$ & $30.0 \%$ & $23.7 \%$ & $100 \%$ \\
\hline Total Corpus & $44.4 \%$ & $27.2 \%$ & $28.2 \%$ & $100 \%$ \\
\hline
\end{tabular}

Tabla 4. Distribución porcentual según propuesta de categorías

\section{REFLEXIONES FINALES}

Tras la identificación de las vacilaciones según su función como pausas oralizadas de conexión (POC), planificadores discursivos (PD) y mecanismos de edición (ME), en los tipos de discurso planificado y no planificado, surgen nuevas observaciones. En primer lugar, se aprecia que las POC (repeticiones más sonidos breves) son un fenómeno sumamente común en cualquier tipo de discurso. Alrededor de 4 de cada 10 fenómenos de vacilación tienen una función de POC. En segundo lugar, las vacilaciones con función ME (frases cortadas más palabras cortadas) son mucho más comunes en discursos de tipo planificado. Se postula que esta tendencia obedece a que los hablantes buscan apegarse al discurso que han elaborado con anticipación, lo que podría provocar una mayor propensión al automonitoreo (Levelt 1983)

21 Incluye semiplanificado. 
y la autocorrección (Cf. Schegloff et al. 1977), que explicarían la mayor aparición de ME. En tercer lugar, las vacilaciones con función PD (sonidos largos más alargamientos de palabra) disminuyen en discursos planificados, precisamente por la naturaleza del discurso, que los requiere aquí en mucha menor proporción.

En contraste con los discursos planificados, en los no planificados se aprecia que los PD se incrementan considerablemente, mientras que los ME disminuyen. Esta tendencia porcentual (ver Tabla 4) ayuda a reforzar el supuesto de que en este caso la ausencia de un esquema, guion o plan discursivo no "compromete" a los hablantes a autocorregirse de manera frecuente, provocando así menos instancias para la producción de ME. Por otro lado, el carácter más espontáneo de estas secuencias ocasionaría el incremento de fenómenos PD: al ser un discurso que se elabora al mismo tiempo en que se reflexiona y que requiere así de la función planificadora otorgada por un elemento de tipo PD.

Tras estas observaciones se podría plantear que ME y PD parecerían encontrarse en "distribución complementaria": el espacio que dejan los ME en discursos no planificados "es ocupado" por los PD, mientras que aquellos espacios dejados por los PD en discursos planificados son tomados por los ME, como se observa en los porcentajes de la Tabla $4^{22}$.

Finalmente, la profundización en el estudio de estos y otros fenómenos de vacilación en este y otros entornos comunicativos, requiere llevar la metodología hacia lo cualitativo. La complementación de los hallazgos cuantitativos con herramientas cualitativas puede dar lugar a investigaciones que ofrezcan explicaciones más acabadas, por ejemplo, un análisis de las vacilaciones y su relación con el estatus o poder del hablante en la situación interactiva, o la relación de estos fenómenos con el tema que está siendo tratado. En esta misma línea, es factible ahondar en estudios desde una perspectiva pragmática: analizar, por ejemplo, los tipos de enunciados como

22 Este tropo no resulta válido si se desagregan los datos y se contrastan de manera independiente discursos planificados, no planificados y semiplanificados. En los no planificados, $24 \%$ son PD y $26.2 \%$ ME, por lo que la explicación no aplica, pues del razonamiento anterior se sigue que el porcentaje de los fenómenos tipo PD tendría que ser más elevado que el de los ME. Sí aplica, sin embargo, para el discurso semiplanificado (correspondiente a las preguntas de los legisladores), donde aparece un $40.2 \%$ de PD y un $19.3 \%$ de ME. Esta aparente contradicción plantea la necesidad de descomponer o analizar con mucho mayor detalle la propia naturaleza discursiva de cada secuencia, sobre todo cuando son tan distintas entre sí. Plantea también la necesidad de ampliar la muestra, así como la necesidad de indagar en otro tipo de interacciones, tanto planificadas como no planificadas, y no necesariamente restringidas al ámbito parlamentario. 
actos de habla, las estrategias de construcción de la imagen de los hablantes, las estrategias de la cortesía verbal y su correlación con la variación en la frecuencia de los distintos fenómenos de vacilación. Para una comunicación posterior, queda el abordaje de las variables rol, sexo, reparación posterior, y repetición del fenómeno, que se mencionan en el §2 y su relación con las vacilaciones etiquetadas en el corpus.

\section{REFERENCIAS BIBLIOGRÁFICAS}

“Ley de la Comisión de Derechos Humanos del Distrito Federal”. 1993. En Diario Oficial de la Federación, 22 de junio de 1993.

“Ley Orgánica de la Asamblea Legislativa del Distrito Federal”. 2008. En Gaceta Oficial del Distrito Federal, 18 de mayo de 2016. [Última reforma, 5 de mayo de 2016]. [En línea] Disponible en http://www.aldf.gob.mx/marco-juridico-102-1.html. [Consulta el 17/8/2016].

Ávila, Silvia. (1999). El análisis del diálogo desde la perspectiva de los estudios de la interacción verbal: los marcadores de vacilación en la entrevista radiofónica. Tesis para optar por al grado de Licenciado en Lengua y Literatura Hispánicas. Facultad de Filosofía y Letras. México, D.F.: UNAM.

Bortfeld, H., Leon, S., J. Bloom, M. Schober, S. Brennan. 2001. "Disfluency rates in conversation: effects of age, relationship, topic, role, and gender". Language and speech 44 (2): 123-147.

Brennan, S. y Williams, M. 1995. "The feeling of another's knowing: prosody and filled pauses as cues to listeners about the metacognitive states of speakers". Journal of Memory and Language 34: 383-398.

Briz, ANTONIO. 1998. El español coloquial en la conversación. Esbozo de pragmagramática. [Edición actualizada noviembre de 2001]. Barcelona: Ariel Lingüística.

Carbó, Teresa. 1987. “Cómo habla el poder legislativo en México?”, Revista Mexicana de Sociología. Año XLIX/Vol. XLIX/ Núm. 2, abril-junio. México, D.F.: UNAM.

Clark, H. H. 1994. "Managing problems in speaking”. En Speech communication, 15, pp. 243-250.

Clark, H. y J. Fox Tree. 2002. "Using uh and um in spontaneous speech". Cognition 84: 73-111.

CRIBLE, L. 2018. Discourse Markers and (Dis)fluency. Forms and functions across languages and registers. Amsterdam/Philadelphia: John Benjamins Publishing Company.

Dalton, P. y W. Hardcastle. 1977. Disorders of fluency. Londres: Edward Arnold.

DuEz, D. 2001. "Signification des hésitations dans la production et la perception de la parole spontanée”. Revue Parole, Vol. 17-19: 113-137. [En línea] Disponible en http://lpl-aix. fr/ fulltext/1198.pdf [Consulta 17/8/2016].

2001b. "Caractéristiques acoustiques et phonétiques des pauses remplies dans la conversations en français". Travaux Interdisciplinaires du Laboratoire Parole et Langage. Vol. 20: 31-48.

Fox Tree, J. 1995. "The effects of false starts and repetitions on the Processing of Subsequent Words in Spontaneous Speech”. Journal of Memory and Language 34: 709-738. 
Goldman-Eisler, F. 1972. "Pauses, clauses, sentences", Language and Speech 15, vol. 2: 103-13.

Instituto Cervantes. 2008. (Antonio Briz, coord.). Saber hablar. México, D.F.: Editorial Aguilar.

JeONG, H. 2011. “¿Vacilar? En español y en coreano”. Actas del I Congreso Ibero-asiático de Hispanistas Siglo de Oro (e Hispanismo general), (Delhi, 9-12 de noviembre, 2010). Vibha Maurya y Mariela Insúa (eds.). Publicaciones digitales del GRISO/Servicio de Publicaciones de la Universidad de Navarra. pp. 327-341.

Kerbrat-Orecchioni, C. 1996. La conversation. París: Éditions du Seuil.

Koch, P. y W. Oesterreicher. 1990. Lengua hablada en la Romania: español, francés, italiano. (Versión española de Araceli López Serena). Madrid: Gredos Biblioteca Románica Hispánica.

LAFOREST, M. 1997. "Hésitation et situation d'interaction: une étude de la variation intra individuelle". Hwave 26. Quebec: Universidad de Laval.

Levelt, W. 1983. "Monitoring and self-repair in speech". Cognition, Vol. 14: 41-104.

LEVInSON, S. C. y F. TorReIRA. 2015. "Timing and turn-taking and its implications for processing models of language". Frontiers in Psychology 6: 731: 1-17.

López Eire, A. 2007. "La naturaleza política y ética de la retórica". Nova Tellvs 25(1): 43-91. UNAM.

MAHL, G. 1987. "Everyday disturbances in spontaneous speech: general properties". Explorations in nonverbal and vocal behaviour, Lawrence Erlbaum Associates Inc., New York, pp. 163-182.

Menuura, M. P. 2007. "La fluidez discursiva oral. Una propuesta de evaluación”. Ogigia. Revista electrónica de estudios hispánicos 1. Enero: 7-16.

Ong, Walter. 1987. Oralidad y escritura. Tecnologías de la palabra. México: Fondo de Cultura Económica.

PRESEEA. 2008. "Marcas y etiquetas mínimas obligatorias". Vers. 1.2. 17-02-2008. Proyecto Para el Estudio Sociolingüístico del Español de España y de América [En línea] Disponible en http://preseea.linguas.net/Portals/0/Metodologia/Marcas_etiquetas minimas_obligatorias_1_2.pdf [Consulta el 18/3/2017].

Quesada, Luis B. 2017. "Los medios, Trump y la fluidez oral”. En LL Journal, Vol. 12, Núm 2 [En línea]. Disponible en https://lijournal.commons.gc.cuny.edu/quesada/ [Consulta el 1/17/2019].

Reynolds, A. y A. Paivio. 1968. "Cognitive and emotional determinants of speech". Canadian Journal of Psychology 22/3: 164-175

Sacks, H., E. Schegloff, G. JefFerson. 1974. "A simplest systematics for the organization of turn-taking for conversation". Language 50: 696-735.

Schachter, Paul. y Timothy Shopen. 2007. "Parts-of-speech systems". Language Typology and Syntactic Description (Timothy Shopen, ed.). New York: Cambridge University Press.

Schachter, Stanley, Nicholas Christenfeld, Bernard Ravina y Frances Bilous. 1991. "Speech disfluency and the structure of knowledge". Journal of personality and social psychology, Vol. 60, núm. 3: 362-367.

Schegloff, E., G. Jefferson y H. Sacks. 1977. "The preference for self-correction in the organization of repair in conversation". Language, Vol. 53, Núm. 2 (Junio): 361-382.

Schober, M. F. 2006. "Dialogue and Interaction". Encyclopedia of Language \& Linguistics, (Keith Brown, ed.). Segunda edición, 3: 564-571. Oxford: Elsevier.

Tannen, Deborah. 1987. "Repetition in conversation: toward a poetics of talk". Language, Vol. 63, núm. 3: 574-602. 
Vasilescu, I., Adda-Decker, M. 2006. "Facteurs caractérisant les hésitations dans les grands corpus: langue, genre, style de parole et compétence linguistique", Actes des XXVI journées d'études sur la parole. Junio de 2006, Dinard.

Vázquez, Laslop, M. 2011. "Elementos del mecanismo de la toma de turnos en el debate parlamentario y alternativas de transcripción", Realismo en el análisis de corpus orales. Primer Coloquio de Cambio y Variación Lingüística (Pedro M. Butragüeño, ed.). México, D.F.: El Colegio de México.

Watanabe, M., K. Hirose, Y. Den y N. Minematsu. 2008. "Filled pauses as cues to the complexity of upcoming phrases for native and non-native listeners". Speech communication 50: 81-94. 